\title{
EL PAPEL DE LA VERDAD EN EL DISCURSO NORMATIVO
}

\author{
Eugenio Bulygin \\ Universidad de Buenos Aires
}

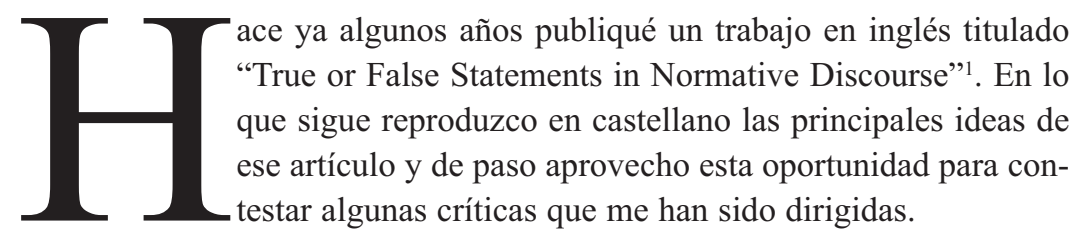

1. El propósito de este trabajo es investigar el papel que desempeña la noción de verdad en el discurso normativo, esto es, tratar de determinar qué oraciones de ese discurso tienen valores de verdad, es decir, son susceptibles de ser verdaderas o falsas. Usaré como hilo conductor la obra de Georg Henrik von Wright, a cuya memoria está dedicado el presente volumen.

Por "discurso normativo" entiendo todo discurso en el que aparecen términos típicamente normativos, como "prohibido", "permitido", "obligatorio", "debe", "no debe", "puede", etc. Oraciones en las que figuran tales términos son llamadas por von Wright "oraciones deónticas". Tales oraciones son frecuentemente usadas para expresar normas, es decir, como formulaciones de normas. Pero es importante tener presente que las normas pueden también ser formuladas mediante otros recursos lingüísticos (imperativos, oraciones en futuro indicativo: vgr. "El que matare a otro será penado...") o no lingüísticos (semáforos, carteles, gestos, etc.).

En su conocido e influyente libro Norm and Action (1963), así como en algunas publicaciones anteriores ${ }^{2}$ (von Wright 1961) von Wright parece distinguir entre cuatro entidades diferentes: formulaciones normativas, nor-

${ }^{1}$ R. Egidi (ed.), In Search of a New Humanism.The Philosophy of Georg Henrik von Wright, Kluwer Dordrecht-Boston-London, 1999, 183-191.

${ }^{2}$ G. H. von Wright, "The Foundation of Norms and Normative Statements" en K. Ajdukiewicx (ed.), The Foundations of Statements and Decisions. Proceedings of the International Colloquium on Methodology of Science, Warsaw 1961, 351-67, reproducido en G.H. von Wright, Practical Reason. Philosophical Papers, vol. I, Oxford 1983, 67-82, citado en lo sucesivo como PR. 
mas, enunciados normativos (normative statements) y proposiciones normativas. Tres de esas entidades son no-lingüísticas y sólo la primera es una entidad lingüística. Las formulaciones normativas son oraciones deónticas usadas para expresar normas; pero también pueden ser usadas para formular enunciados normativos. Cuando una oración deóntica es usada prescriptivamente expresa una norma (que no es ni verdadera ni falsa); cuando es usada descriptivamente, expresa un enunciado normativo que es verdadero o falso. Las proposiciones normativas también son verdaderas o falsas. Tenemos pues dos entidades que admiten valores de verdad: enunciados normativos y proposiciones normativas. Pero no es muy claro qué son los enunciados normativos y cómo están relacionados con las proposiciones normativas.

"Un enunciado normativo... es un enunciado que dice que algo debe, puede o no debe ser hecho (por un agente o agentes, en alguna ocasión o en general, incondicionalmente o cuando están dadas ciertas condiciones). El término "enunciado" [statement] es usado aquí en un sentido que propongo llamar "estricto". Un enunciado en sentido estricto es verdadero o falso. (La oración que es usada para hacer un enunciado expresa una proposición).” (N\&A, 105)

Por otro lado, una proposición normativa es definida como una proposición que dice que una norma existe ("La proposición de que existe tal o cual norma será llamada proposición normativa" N\&A, 106).

Estas citas sugieren la siguiente distinción: Una oración usada (descriptivamente) para decir que una acción es obligatoria, está prohibida o permitida (i.e. que algo debe, puede o no debe ser hecho) expresa un enunciado normativo, mientras que una oración usada para decir que una determinada norma existe expresa una proposición normativa. Para usar un ejemplo (levemente modificado) de von Wright: Si en respuesta a una pregunta digo "Vd. no debe estacionar su coche frente a mi casa" y lo que estoy haciendo no es prohibir, sino darle al que pregunta una información respecto de las reglas de estacionamiento, entonces mi oración es descriptiva y el enunciado que hice es un enunciado normativo. Si, en cambio, digo "Hay una norma que prohibe estacionar en este lugar", entonces mi oración expresa una proposición normativa.

Pero esta sugerencia no parece ser aceptable. ¿Cuál es la diferencia entre informar sobre las reglas de estacionamiento existentes y decir que hay una norma que prohibe estacionar? ¿Cómo podría yo informar sobre las regulaciones existentes, sino diciendo que hay una norma que prohíbe estacionar? Si mi información es verdadera, entonces hay efectivamente una norma que prohibir estacionar y si no hay tal norma mi enunciado normativo es falso. Contrariamente a las apariencias, las dos oraciones dicen lo mismo. En otras palabras, aunque el enunciado normativo parece referirse a la acción de estacionar y la proposición normativa parece referirse a la 
existencia de una norma, tanto uno como la otra tienen las mismas condiciones de verdad. ${ }^{3}$

Pero entonces no tenemos tres, sino dos entidades extra-lingüísticas: normas y proposiciones normativas. Lo que von Wright llama "enunciados normativos" no son más que proposiciones normativas disfrazadas. Las oraciones

(1) Vd. no debe estacionar aquí

y

(2) Hay una norma que prohibe estacionar aquí

dicen lo mismo. Las oraciones deónticas usadas descriptivamente pueden adoptar dos formas, una forma elíptica y una forma expandida. En su forma elíptica dicen que una acción p (o un estado de cosas resultante de una acción) está prohibida (permitida o es obligatoria); en su forma expandida, dicen que hay una norma que prohíbe (permite, obliga) hacer p.

La forma elíptica es lo que Carnap alguna vez llamó "the transposed mode of speech":

By a transposed mode of speech we mean one in which, in order to assert something about an object $a$, something corresponding is asserted about an object $b$ which stands in a certain relation to the object $a^{4}{ }^{4}$

En su forma expandida la proposición normativa dice que hay una norma (objeto $a$ ) que prohibe o permite la acción p. En vez de esto, en el modo traspuesto decimos que la acción p (objeto $b$ ) está prohibida o permitida. Esto no es más que la forma elíptica de la misma proposición normativa.

En ambos casos, la proposición normativa es relativa a una norma o un conjunto de normas, si bien tal referencia no siempre es explícita. Una oración como (2) que expresa una proposición normativa en su forma expandida tiene la ventaja de no ser ya ambigua. Ella no podría ser usada para emitir una prohibición, pues es claramente descriptiva, mientras que la oración (1) es característicamente ambigua, pues se usa tanto para formular una norma, como para expresar una proposición normativa.

Esta interpretación que equipara los enunciados normativos con las proposiciones normativas se apoya también en el hecho de que en las publicaciones posteriores von Wright deja de usar la expresión "enunciado normativo" y sólo habla de normas y de proposiciones normativas.

Pero por otro lado, si los enunciados normativos sólo son una forma elíptica de las proposiciones normativas, pierde todo sentido hablar acerca del

\footnotetext{
${ }^{3}$ En el artículo de 1999 dije, además, que tenían el mismo significado. Esto último es probablemente una exageración, que no estoy dispuesto a mantener. En todo caso, en mi artículo no di ninguna razón para sostener esta afirmación.

${ }^{4}$ R. Carnap, The Logical Syntax of Language, Paterson, New Jersey, 1959, 308 (primera edición en inglés, Londres, 1937).
} 
fundamento de los enunciados normativos. Este tema recibe su desarrollo en el artículo titulado precisamente "The Foundation of Norms and Normative Statements" (1961) y es mencionado en Norm and Action: "Por fundamento de la verdad de un enunciado normativo dado entiendo la respuesta verdadera a la pregunta por qué la cosa en cuestión debe, puede o no debe ser hecha" (N\&A, 105). Curiosamente, aunque en publicaciones posteriores a N\&A ya no habla de enunciados normativos ${ }^{5}$, el artículo de 1961 ha sido incluido por von Wright en su libro Practical Reason (1983).

Ahora bien, si la proposición expresada en un enunciado normativo es simplemente una proposición normativa, entonces la pregunta acerca del por qué parece estar fuera de lugar. Normalmente, uno no pregunta "¿por qué? frente a una proposición descriptiva. Si por ejemplo digo "La nieve es blanca" y alguien me pregunta “¿Por qué?”, me sentiría perplejo; no sabría qué contestar. La respuesta "Porque es así" no parece ser muy ilustrativa y lo mismo vale para otras respuestas: "Porque la naturaleza es así" o "Porque Dios ha creado las cosas de esta manera". Y, sin embargo, von Wright dice enfáticamente:

"Un importante tipo de respuesta a la pregunta'¿Por qué debe (puede, no debe) hacerse tal o cual cosa?" es el siguiente. Hay una norma que prescribe que esto debe (puede, no debe) hacerse. La existencia de una norma es, aquí, el fundamento o razón de verdad del enunciado normativo. (PR,68).

No veo por qué una respuesta tal deba considerarse importante, pues equivale a la repetición -en forma expandida- de la misma proposición normativa ya expresada en el enunciado normativo. Me parece que en este caso von Wright ha sido víctima de la ambigüedad sistemática de las oraciones deónticas que fue denunciada enfáticamente por él. Esto porque la pregunta “¿Por qué?” y la respuesta "Porque hay una norma...” sólo tienen sentido frente a oraciones deónticas usadas para prescribir, es decir, frente a las que expresan normas. En tal caso es perfectamente razonable contestar "Vd. debe hacer p porque hay una norma que prescribe p", aunque aún así la respuesta no es del todo satisfactoria, a menos que se agregue "y Vd. debe obedecer esta norma" (lo que normalmente es presupuesto tácitamente).

Si esto se acepta, entonces no hay tal cosa como "el problema filosófico del fundamento de los enunciados normativos" (PR, 67).

2. En un muy interesante trabajo ${ }^{6}$ Pablo Navarro formula algunas críticas a mi artículo de 1999. Como ya señalé en la nota 2, acepto la crítica dirigida

${ }^{5}$ Así por ejemplo en "Norms, Truth and Logic" de 1982 dice: "En Norm and Action (1963) hice una distinción tripartita que creo útil entre normas, formulaciones normativas y proposiciones normativas". Como hemos visto esto no es del todo cierto. Pero en todo caso, los enunciados normativos no son mencionados.

${ }^{6}$ Pablo E. Navarro, "Legal Reasoning and Systematization of Law" en A. Soeteman (ed.) Pluralism and Law, Kluwer, Dordrecht-Boston-London, 2001, 251-277. 
a mi tesis de identidad de sentido entre las oraciones (1) y (2), aunque estoy dispuesto a mantener su equivalencia material, es decir, la igualdad de sus condiciones de verdad. Pero Navarro me atribuye una tesis que nunca he formulado, y que él llama concepción reduccionista que, según Navarro, consiste en suponer que "las normas jurídicas existen sólo en la medida en que pertenecen a un sistema jurídico, esto es, su pertenencia a un sistema jurídico es el único hecho que hace verdaderas a las proposiciones normativas."

Dije y repito que nunca he sostenido esta tesis. El término existencia referido a normas está lejos de ser unívoco; en diferentes contextos se usan distintos conceptos de existencia. La enumeración que hace Navarro en la p. 257 de su ensayo lo muestra claramente y no es casualidad que esta lista de los distintos modos de existencia de normas proviene de algunas publicaciones mías. Por lo tanto, la afirmación categórica de Navarro: "We should remember that the reductive conception assumes that a norm exists only to the extent that it belongs to a normative system." (Navarro, op. cit. 258) es ciertamente incorrecta. Lo que he dicho y estoy dispuesto a sostener es que el hecho de que una acción sea obligatoria no puede sino provenir de una norma. La existencia de una norma es condición necesaria para que una acción pueda ser calificada de obligatoria. Pero esto no quiere decir que la pertenencia sea el único hecho que hace verdadera a una proposición normativa. La cosa puede ser mucho más compleja. Yo puedo estar obligado a realizar la acción $\mathrm{p}$, no porque haya una norma en el sistema jurídico en cuestión que me obligue a hacerlo, sino porque el sistema jurídico remite a otro sistema, donde sí hay una norma que me obliga. Una norma perteneciente al sistema puede no ser aplicable (como en el ejemplo de vacatio legis) y una norma que no pertenece al sistema jurídico en cuestión puede resultar aplicable y hacer verdadera la correspondiente proposición jurídica. Esto ocurre en ciertas condiciones con las normas derogadas. ${ }^{8}$

3. En una de sus últimas contribuciones a la lógica deóntica ${ }^{9}$ von Wright introduce una nueva categoría de oraciones normativas susceptibles de ser verdaderas o falsas, que llama oraciones de necesidad práctica. Si bien tales oraciones están mencionadas en sus obras anteriores, es en este artículo en el que von Wright les asigna un papel muy importante en el discurso normativo:

\footnotetext{
${ }^{7}$ Navarro 2001, 256.

${ }^{8}$ Cfr. E. Bulygin, “Tiempo y Validez” en C. Alchourrón y E. Bulygin, Análisis Lógico y Derecho, Madrid 1991, 195-214.

9 "Ought to be - Ought to do" en Six Essays in Philosophical Logic, Acta Philosophica Fennica, Helsinki, 1996, 63-70.
} 
"Otro servicio que la distinción [entre normas y necesidades prácticas] presta a esta materia es que asigna a la noción de verdad un papel en los contextos normativos mucho más grande que algunos filósofos, incluyendo a mí mismo, solían pensar en el pasado. No sólo las proposiciones existenciales ("proposiciones normativas") que dicen que tal y tal norma existe (fue dictada) son verdaderas o falsas, sino también los enunciados acerca de lo que tiene que ser o puede ser hecho a los efectos de seguir lo que ella regula. (von Wright 1996, 70)".

Me parece muy importante la distinción trazada por von Wright entre normas y necesidades prácticas, como es importante su distinción entre dos tipos de normas que von Wright denomina "Tun-Sollen" y "Sein-Sollen types" (tipo de deber hacer y tipo de deber ser). El primer tipo requiere la realización de una o varias acciones; el segundo, requiere que se dé un estado de cosas. Pero decir que el primer tipo es descriptivo y sólo el "Sein-Sollen type" es prescriptivo (p. 69) me parece una exageración. No es demasiado difícil encontrar ejemplos de normas del tipo de deber hacer. Órdenes como “¡Tírese al agua!” o “¡Coma esta sopa!” son claramente de tipo de deber hacer, puesto que imponen la obligación de hacer, es decir, de realizar una cierta acción. Lo que estas normas prescriben es una acción y no un estado de cosas. Por lo tanto, no es verdad que sólo las normas que se refieren a estados de cosas son prescriptivas. Me inclino a pensar que hay normas genuinas de ambos tipos.

En segundo lugar, no siempre es fácil distinguir entre una norma que impone una obligación de realizar una acción y una norma que impone la obligación de producir (o mantener) un estado de cosas. La orden dada a la mucama "Por favor, ;prepare la cena para antes de las 8!" puede ser interpretada como una norma del tipo de "deber ser" (en cuyo caso la mucama está obligada a lograr que se dé un estado de cosas, a saber, que la cena esté lista antes de las 8) o como una norma del tipo de "deber hacer", en cuyo caso la mucama tiene la obligación de realizar una acción (preparar la cena antes de las 8). Preparar una cena es una acción muy compleja que requiere la realización de muchas otras acciones, pero una acción compleja es una acción y no un estado de cosas.

En tercer lugar, no es nada claro cuál es el criterio para distinguir entre lo que uno debe hacer y lo que tiene que hacer para obedecer o satisfacer una norma, es decir, entre una genuina obligación y una necesidad práctica. El uso lingüístico es, como observa von Wright, más bien vacilante, pues expresiones como "debe", "tiene que" o "ha de" son usadas en ambos contextos: en contexto normativo y en contexto práctico.

Supongamos que se ha ordenado a A abrir una cierta ventana; A esta sentado y a menos que se levante no puede abrir la ventana. Su acción de levantarse es una condición necesaria para su acción de abrir la ventana. ¿Diríamos que A debe levantarse (obligación) o que tiene que levantarse 
(necesidad práctica para cumplir con la orden)? Me inclino a pensar que A no tiene la obligación de levantarse, pero si quiere obedecer la orden, tiene que levantarse. En tal supuesto éste sería un caso de necesidad práctica y la oración "A tiene que levantarse" sería verdadera con relación a la orden. Supongamos ahora que la condición no sólo es necesaria por razones empíricas, sino que es lógicamente necesaria. Tomemos el caso de A quien se le ha ordenado tocar piano, pero está prohibido fumar en el lugar donde debe hacerlo. ¿Tiene la obligación de tocar el piano sin fumar? Me siento inclinado a contestar afirmativamente. Obviamente, A tiene que tocar el piano sin fumar. Pero ¿diríamos que tiene una obligación en virtud de las dos normas o que es una necesidad práctica para él de hacer ambas cosas (esto es, tocar piano y abstenerse de fumar) si quiere obedecer ambas normas? Suena raro decir que A tiene que tocar el piano sin fumar, pero que él no tiene ninguna obligación de actuar de esta manera. Más aún, conforme al criterio de von Wright las normas Op y Oq implican la norma O(p.q), pues el conjunto de las dos primeras normas es inconsistente si le agregamos la norma-negación de $\mathrm{O}($ p.q), que es P-(p.q). El conjunto formado por $\{\mathrm{Op}, \mathrm{Oq}, \mathrm{P}-(\mathrm{p} . \mathrm{q})\}$ es inconsistente, porque la conjunción de p.q.-(p.q) es irrealizable, pues se trata de una contradicción. Y si la norma (Op.q) es implicada por las normas Op y Oq, entonces la acción de A consistente en tocar el piano sin fumar es una genuina obligación y no una mera necesidad práctica. La generalización de este ejemplo nos llevaría a lo que cabe llamar el Principio de Obligatoriedad: Es obligatorio (conforme a un conjunto de normas dado) realizar todas las acciones que son lógicamente necesarias para satisfacer todas las obligaciones establecidas por las normas de ese conjunto. Este resultado suena un tanto extraño. Si una acción es empíricamente necesaria, entonces no hay obligación, sino mera necesidad práctica. Pero si es lógicamente necesaria, entonces hay una obligación genuina. Pienso que necesitamos una explicación de esta diferencia. 